\title{
Família, Pares e Delinquência Juvenil: Análise de Diferentes Percursos de Reincidência
}

\author{
Family, Peers and Juvenile Delinquency: \\ Analysis of Different Pathways to Recidivism \\ Cláudia Cunha, Mónica Soares, Lurdes Veríssimo, \& Raquel Matos \\ Universidade Católica Portuguesa, Porto, Portugal
}

\begin{abstract}
Resumo: Diversas dimensões relativas à família, como a estrutura e as dinâmicas familiares (cf., Contreras, Molina \& Cano, 2011), e relacionadas com o grupo de pares (cf. Cottle, Lee \& Heilbrun, 2001) têm sido associadas à reincidência de comportamentos delinquentes em jovens. Trazendo novos contributos para esta discussão, o objetivo do estudo apresentado consiste em avaliar como diferentes dimensões de risco familiares e do grupo de pares variam de acordo com diferentes percursos de reincidência (i.e., não reincidentes, reincidentes intermédios, reincidentes persistentes). Recorrendo a uma amostra de 105 jovens em cumprimento de medida tutelar educativa, este estudo foi realizado em Portugal e centrou-se na reincidência dos jovens no contacto com o sistema de justiça como indicador de percursos delinquentes persistentes. Os resultados revelam que mais de metade dos jovens da amostra (54.3\%) são reincidentes, tendo cumprido medidas tutelares educativas anteriores. Entre as variáveis significativamente associadas à reincidência, destacam-se a existência de contactos anteriores com o sistema de proteção e os comportamentos delinquentes do grupo de pares. Em conjunto, esses fatores parecem relacionar-se com o envolvimento em atos delinquentes, enfatizando a necessidade de serem desenvolvidas intervenções integradas para prevenir a reincidência.
\end{abstract}

Palavras-chave: medidas tutelares educativas, reincidência, fatores de risco, família, grupo de pares.
Abstract: Different family factors such as structure, family dynamics (cf. Contreras, Molina \& Cano, 2011) and peer-related variables (cf. Cottle, Lee \& Heilbrun, 2001) have been linked to recidivism among young offenders. Bringing new contributions to this discussion, our goal was to evaluate how different family and peer factors of risk differ according to various recidivism pathways (e.g., non-reoffenders; moderate reoffenders; persistent reoffenders). Taking a sample of 105 young offenders, complying with youth justice measures, the present study has been developed in Portugal and focuses on recidivism as an indicator of persistent reoffending. Results showed that more than half of young offenders in the sample $(54.3 \%)$ are reoffenders, as they have already complied with former youth justice measures. Among the most relevant variables associated with recidivism are a previous connection with the protection system, and the criminal behavior of the young offenders' peers. Together, these factors may relate to delinquent behavior, highlighting the need to develop integrated interventions, in order to prevent recidivism.

Keywords: youth justice measures, recidivism, risk factors, family, peers.

Um agradecimento especial aos jovens que aceitaram participar neste estudo, às suas famílias e seus representantes legais. Agradecemos também à Direção Geral de Reinserção e Serviços Prisionais, por possibilitar a recolha de dados, e à Comissão Europeia, entidade financiadora do Projeto em que este estudo se enquadra ("Project MERLINO - Towards a juvenile delinquency prevention policy: A multi-state experiment on integration and effi-cacy", HOME/2011/ISEC/AG/4000002611).

Contato: R. Matos. Rua de Diogo Botelho, No 1327|4169-005, Porto, Portugal. Correio electrónico: rmatos@porto.ucp.pt

Como citar: Cunha, C., Soares, M., Veríssimo, L., \& Matos, R. (2015). Família, pares e delinquência juvenil: Análise de diferentes percursos de reincidência. Revista de Psicología, 24(2), 1-18.

http://dx.doi.org/10.5354/0719-0581.2015.38134 


\section{Introdução}

A Lei Tutelar Educativa (Lei No $166 / 99$ de 14 de Setembro) ${ }^{1}$ constitui, em Portugal, o quadro de referência da intervenção na delinquência juvenil, definida como "prática, por menor com idade compreendida entre os 12 e os 16 anos, de facto qualificado na lei como crime [que] dá lugar à aplicação de medida tutelar educativa" (1999, p. 6320). Em concreto, esta Lei estabelece um conjunto de medidas, institucionais (em regime de internamento) e não institucionais (na comunidade), que procuram responsabilizar e "educar para o Direito" (1999, p. 6321) jovens que adotam comportamentos delinquentes. Dados da Direção Geral de Reinserção e Serviços Prisionais (DGRSP, 2014), a entidade responsável pela implementação e monitorização das medidas tutelares educativas em Portugal, apontam para 1,404 medidas em execução em outubro de 2014, com destaque para a medida de acompanhamento educativo e programas formativos $(46 \%)$.

A eficácia das medidas na prevenção da reincidência nos comportamentos delinquentes exige não apenas uma aplicação e monitorização adequada, mas também uma intervenção em diferentes dimensões da vida dos jovens (cf. Sousa Santos, 2004). Para tal, é importante conhecer aprofundadamente os percursos de vida dos jovens sinalizados por delinquência, em dimensões como a família ou o grupo de pares, contributo ainda escasso na literatura nacional sobre delinquência juvenil. Esse é o principal foco do estudo empírico que se apresenta neste artigo.

\footnotetext{
${ }^{1}$ Alterada pela Lei $\mathrm{N}^{\mathrm{o}}$ 4/2015 de 15 de Janeiro.
}

\section{Reincidência em Percursos Delinquen- tes Persistentes: Contributos da Abor- dagem Desenvolvimental}

Orientada para a compreensão de problemas de comportamento apresentados pelos jovens ao longo do seu desenvolvimento, a abordagem desenvolvimental privilegia o estudo de manifestações antissociais precoces e de manifestações exibidas na adolescência e entendidas como delinquentes (Dishion \& Patterson, 2006). Considera-se ainda que uma série de fatores individuais, familiares, relacionais e contextuais podem afetar a propensão para um jovem exibir comportamentos delinquentes, e que estes fatores têm um impacto diferenciado em função da fase desenvolvimental em que exercem a sua influência (Shader, 2003) ${ }^{2}$.

Enquadrados nesta abordagem, e centrados tanto no autorrelato da delinquência como em registos oficiais, vários estudos têm analisado quais as especificidades das trajetórias de delinquência persistente (Andersson, Levander, Svensson, \& Levander, 2012; Le Blanc \& Fréchette, 1989; Moffit, 1993), bem como os fatores de risco e de proteção ${ }^{3}$ que lhes estão associados (e.g., Chung \& Steinberg, 2006; Stouthamer-Loeber, Loeber, Wei, Farrington, \& Wikström, 2002; Stouthamer-

\footnotetext{
${ }^{2}$ De entre os fatores destacados na literatura, o estudo empírico que se apresenta centra-se nos familiares e do grupo de pares.

${ }^{3}$ Qualquer dimensão, passível de ser identificada na trajetória de um jovem, que aumente a probabilidade de ele adotar comportamentos delinquentes poderá ser entendida como um fator de risco (Farrington, Loeber, \& Ttofi, 2012; Day, Wanklyn, \& Yessine, 2014). Por sua vez, um fator protetor pode ser entendido como uma dimensão que atenua a probabilidade de um jovem em risco se envolver em ofensas (efeito promotor) ou como uma variável que interage com um fator de risco, mitigando ou anulando o seu impacto (efeito interativo) (Farrington \& Ttofi, 2011).
} 
Loeber, Loeber, Stallings, \& Lacourse, 2008). A este nível, destacam-se dois argumentos-chave empiricamente validados. Em primeiro lugar, o comportamento delinquente persistente tende a iniciar durante a infância (Le Blanc \& Fréchette, 1989; Kazemian \& Farrington, 2005; Moffitt, 1993; Van Domburgh, Loeber, Bezemer, Stallings, \& Stouthamer-Loeber, 2009). Nesta linha de análise, vários autores propõem trajetórias-tipo em que a persistência de comportamentos delinquentes é geralmente definida pela sua precocidade e estabilidade ao longo da vida (Andersson et al., 2012; Fréchette \& Le Blanc, 1987; Moffitt, 1993, 2006). Em segundo lugar, quanto maior a acumulação de fatores de risco e menor o número de fatores protetores na trajetória desenvolvimental de um jovem, maior a probabilidade para o seu envolvimento em comportamentos delinquentes de caráter grave e persistente (Stouthamer-Loeber et al., 2002).

Privilegiando os contactos com o sistema de justiça como indicadores de comportamentos delinquentes persistentes, este estudo pretende avaliar como dimensões de risco, familiares e do grupo de pares, se apresentam em trajetórias de jovens com diferentes percursos de reincidência.

\section{Família}

Mesmo assumindo que durante a adolescência, o valor preditivo de variáveis relativas à família poderá ser menor do que o de variáveis associadas ao grupo de pares (Aseltine, 1995; Hoeve et al., 2009; Thornberry, Krohn, Lizotte, Smith, \& Porter, 1998), diversas dimensões familiares (e.g. estrutura familiar, história criminal de familiares) parecem relacionar-se com a reincidência de comportamentos delinquentes em jovens com história de ofensas registadas pelo sistema de justiça
(Contreras et al., 2011). Três fatores familiares serão analisados neste estudo: a história criminal da família, as práticas educativas parentais e a experiência de vitimação.

História Criminal da Família. A história criminal da família parece ser um fator determinante na compreensão das trajetórias dos jovens que já exibiram comportamentos delinquentes ao longo da vida. O background familiar, avaliado a nível dos contactos anteriores com o sistema de justiça e do uso de drogas, pode ser um preditor relevante da delinquência juvenil e da reincidência "criminal" entre os jovens, ao contribuir para uma maior legitimação dos comportamentos delinquentes (Ang \& Huan, 2008; Huizinga, Weiher, Menard, Espiritu, \& Esbensen, 1998). Se os delitos perpetrados no seio familiar de origem resultarem numa condenação de pais ou irmãos, este fator surge como o preditor mais forte de comportamentos antissociais e ofensivos persistentes cometidos pelos jovens ao longo do seu percurso desenvolvimental (Farrington, 2003; Murray \& Farrington, 2005).

Práticas Educativas Parentais. De forma geral, práticas educativas caracterizadas como excessivamente punitivas, inconsistentes ou negligentes, ou, em contraste, como demasiado passivas ou permissivas, podem predizer comportamentos delinquentes (Farrington et al., 2012). A punição física recorrente, por exemplo, enquadrada num estilo educativo autoritário, tem sido associada a um risco superior para o jovem delinquir (Loeber, Farrington, Stouthamer-Loeber, Moffitt, \& Caspi, 1998). No entanto, essa associação não é consensual, sendo também argumentado que essa associação é residual, impondo-se uma análise mais ampla da relação entre problemas de comportamen- 
to e punição física (Morris \& Gibson, 2011). Outros estudos mostram ainda que a punição física pode, na perspetiva de jovens ofensores, assumir um papel disciplinador e dissuasor na adoção de comportamentos delinquentes (Simões, Matos, \& Batista-Foguet, 2008). De outra forma, as práticas educativas negligentes associam-se à perpetração de comportamentos delinquentes (Lee, Onifade, Teasley, \& Noel, 2012) e parecem predizer as carreiras criminais persistentes (Hoeve et al., 2007; Mulder, Vermunt, Brand, Bullens, \& Van Marle, 2012). Essas práticas educativas, segundo Farrington (2003), parecem assumir-se como estáveis ao longo da trajetória desenvolvimental, o que prossupõe a sua relevância na manutenção de comportamentos delinquentes. Pelo contrário, práticas educativas consistentes, adequadas e assentes na disciplina positiva assumem-se como protetoras em termos da adoção de comportamentos delinquentes (Farrington \& Ttofi, 2011).

Diversos estudos sobre práticas educativas parentais e risco de delinquência juvenil centram-se em particular na monitorização parental, respeitante ao grau de controle e vigilância exercido pelos pais (Farrington, 2010). A monitorização parental tem sido definida por alguns autores a partir de três dimensões: a solicitação de informações por parte dos pais, o estabelecimento de regras e limites e, por último, a confiança dos filhos em revelarem aos pais informações sobre as suas rotinas e paradeiro (Stattin \& Kerr, 2000). Uma monitorização parental pobre parece ser um dos preditores mais significativos no que diz respeito à predisposição para a violação de normas (Stattin \& Kerr, 2000) e para a adoção de comportamentos delinquentes pelos jovens (Hoeve et al., 2009). Em contrapartida, quando os pais monitorizam as atividades dos seus filhos, há um efeito protetor (Griffin,
Botvin, Scheier, Diaz, \& Miller, 2000; Huizinga et al., 1998), principalmente em fases precoces da adolescência (Hartinger-Saunders, Rine, Wieczorek, \& Nochajski, 2012).

Diferentes indicadores da monitorização parental serão avaliados neste estudo (cf. Stattin \& Kerr, 2000): a frequência de tempo que os jovens passam com os pais, as práticas educativas específicas (e.g. punição física) e o número de irmãos do jovem, assumindo que há menor capacidade para gerir e supervisionar o comportamento dos filhos, quando o seu número é elevado (Farrington, 2010; Ireland, Smith \& Thornberry, 2002).

Experiência de Vitimação. A experiência de vitimação na infância, nomeadamente ao nível familiar, tem sido apontada como um fator preditor de comportamentos delinquentes posteriores (Crooks, Scott, Wolfe, Chiodo, \& Kilip, 2007; Farrington, 2010; Smith \& Thornberry, 1995). Experiências de vitimação na adolescência podem também ser relevantes para compreender a emergência de comportamentos delinquentes. Ireland e colaboradores (2002) evidenciaram que experiências de vitimação na adolescência (entre os 12 e os 17 anos) predizem comportamentos delinquentes perpetrados pelos jovens durante este mesmo período.

De modo geral, o maltrato tem sido correlacionado com o maior número de detenções na idade adulta (Smith, Ireland, \& Thornberry, 2005). Contudo, a nível da reincidência nos contactos estabelecidos com o sistema de justiça entre jovens, pouco ainda foi discutido relativamente ao papel da experiência de vitimação. Um dos escassos contributos é o de Stewart, Dennison e Waterson (2002), que verificaram através de um estudo longitudinal que, entre os jovens com contactos com o 
sistema de justiça, a probabilidade de iniciar comportamentos delinquentes precocemente e com maior gravidade é superior nos que têm também contactos anteriores com o sistema de proteção. Um estudo mais recente evidenciou também que quando os jovens estão envolvidos em ambos os sistemas de justiça e de proteção, apresentam maior probabilidade de reincidência criminal, mesmo quando outras variáveis, individuais, escolares, familiares e do grupo de pares, são controladas (Ryan, Williams, \& Courtney, 2013).

Em Portugal, o sistema de proteção assenta na Lei de Promoção e Proteção de Crianças e Jovens em Risco (Lei N ${ }^{\circ}$ 147/99 de 1 de Setembro), direcionada para a intervenção junto de menores cujos pais ou representantes legais coloquem em perigo a sua segurança, saúde, formação, educação ou desenvolvimento, tanto por via da ação como da omissão. Desta forma, um processo de promoção e proteção é iniciado quando uma criança ou jovem experiencia maltrato ativo ou passivo. A existência de um processo desta natureza apresenta-se assim como um indicador formal de experiências de vitimação sofridas por crianças e jovens.

\section{Grupo de Pares}

As relações com pares assumem-se como um contexto desenvolvimental relevante na compreensão de diversos comportamentos, laços afetivos e objetivos estabelecidos pelos jovens ao longo do seu desenvolvimento (Vitaro, Brendgen, \& Wanner, 2005). Devido ao seu papel socializador incontornável durante a adolescência, o grupo de pares pode oferecer o contexto para o uso de normas que regulam os próprios comportamentos delinquentes e violentos (Haynie \& Payne, 2006). Assim, a adoção de comportamen- tos de risco pode ser fortemente influenciada pelo grupo de pares (Gardner \& Steinberg, 2005).

Em particular, quando percecionado como desviante ou delinquente, o grupo de pares pode ser conceptualizado como uma dimensão de risco para a delinquência juvenil (Church II et al., 2012; Fergusson, Vitaro, Wanner, \& Brendgen, 2007; Huizinga et al., 1998; Içli \& Çoban, 2012; Lee et al., 2012; Simões et al., 2008), principalmente durante a adolescência, altura em que o processo de socialização passa a estar sobretudo associado ao grupo de pares (Brown, 1990 cit em Haynie \& Payne, 2006). Em conformidade, dados empíricos demonstram que durante a adolescência a interação com pares com comportamentos desviantes é preditora do comportamento delinquente (Vitaro et al., 2005). Alguns autores (e.g. Aseltine, 1995; Fergusson et al., 2007; Huizinga et al., 1998; Simões et al., 2008) apontam mesmo que a adoção de comportamentos delinquentes por parte do grupo de pares é a dimensão preditiva da delinquência juvenil com maior peso e impacto, correlacionando-se igualmente com a "reincidência" criminal (Cottle et al., 2001).

A literatura demonstra ainda que os jovens tendem a reproduzir o mesmo tipo de comportamentos desviantes, nomeadamente roubos e consumos de drogas, exibido pelos seus pares (Thomas, 2015). Por exemplo, um estudo conduzido recentemente na Turquia mostra como a probabilidade de cometer um roubo duplica entre os jovens com pares que já o fizeram (cf. Içli \& Çoban, 2012).

\section{Objetivos}

O presente estudo centra-se em dimensões da família e do grupo de pares em jovens que se diferenciam pelo padrão de reinci- 
dência nos contactos com o sistema de justiça. Os seus objetivos específicos são: Caracterizar o percurso judicial de jovens em cumprimento de medida tutelar educativa segundo o tipo de crime cometido, o tipo de medida tutelar educativa atual, a presença de medidas tutelares educativas anteriores, a precocidade da aplicação primeira medida tutelar educativa e a reincidência; Descrever e compreender como variáveis da família e do grupo de pares se associam a diferentes percursos de reincidência.

\section{Método}

\section{Amostra}

A amostra é constituída por 105 jovens a cumprir medida ao abrigo da Lei Tutelar Educativa (Lei № 166/99 de 14 de Setembro $^{4}$ ), com idades compreendidas entre os 14 e os 17 anos) $)^{5}$. No total, $86.7 \%$ dos jovens são do sexo masculino e $13.3 \%$ do sexo feminino. A média de idades dos participantes é de $15.89(D P=0.98)$. Através da consulta da tabela 1 , verifica-se que, na sua maioria, os participantes residem na zona metropolitana de Lisboa ( $n=84$; $80.0 \%$ ), seguindo-se a zona metropolitana do Porto $(n=7 ; 6.7 \%)$. Relativamente ao nível de escolaridade, salientamos que 21 jovens estão a frequentar o $6^{\circ}$ ano $(20.0 \%)$ e 45 estão a frequentar $9^{\circ}$ ano $(42.9 \%)$. A tipologia de ensino mais comum é o ensino técnico $(n=56 ; 53.3 \%)$. A nível das retenções escolares, apenas $5.7 \%$ dos jovens nunca ficaram retidos. Em contrapartida, 47 jovens já ficaram retidos mais de três vezes (44.8\%). Apesar de não constar nos dados apresentados na Tabela 1, importa acrescentar que cerca de $38.0 \%$ dos jovens da amostra já estiveram ou estão em situação de abandono escolar.

\footnotetext{
${ }^{4}$ Alterada pela Lei $\mathrm{N}^{\mathrm{o}}$ 4/2015 de 15 de Janeiro.

${ }^{5}$ Estes foram os dois critérios de inclusão que nortearam a constituição da amostra.
}

Tabela 1

\section{Caracterização Descritiva da Amostra}

\begin{tabular}{|c|c|c|}
\hline & $n$ & $\%$ \\
\hline \multicolumn{3}{|l|}{ Sexo } \\
\hline Feminino & 14 & $13.3 \%$ \\
\hline Masculino & 91 & $86.7 \%$ \\
\hline \multicolumn{3}{|l|}{ Idade } \\
\hline 14 & 10 & $9.5 \%$ \\
\hline 15 & 27 & $25.7 \%$ \\
\hline 16 & 33 & $31.4 \%$ \\
\hline 17 & 35 & $33.3 \%$ \\
\hline \multicolumn{3}{|l|}{ Nacionalidade } \\
\hline Portuguesa & 92 & $87.6 \%$ \\
\hline Angolana ou guineense & 7 & $6.7 \%$ \\
\hline Outras nacionalidades & 4 & $3.8 \%$ \\
\hline Valor em falta & 2 & $1.9 \%$ \\
\hline \multicolumn{3}{|l|}{ Área de residência } \\
\hline Lisboa & 84 & $80.0 \%$ \\
\hline Porto & 7 & $6.7 \%$ \\
\hline Setúbal & 2 & $1.9 \%$ \\
\hline Outros & 11 & $10.5 \%$ \\
\hline Valor em falta & 1 & $1 \%$ \\
\hline \multicolumn{3}{|l|}{ Ano de escolaridade } \\
\hline $5^{\circ}$ ano & 2 & $1.9 \%$ \\
\hline $6^{\circ}$ ano & 21 & $20.0 \%$ \\
\hline $7^{\circ}$ ano & 11 & $10.5 \%$ \\
\hline $8^{\circ}$ ano & 3 & $2.9 \%$ \\
\hline $9^{\circ}$ ano & 45 & $42.9 \%$ \\
\hline $10^{\circ}$ ano & 7 & $6.7 \%$ \\
\hline $11^{\circ}$ ano & 0 & $0.0 \%$ \\
\hline $12^{\circ}$ ano & 1 & $1.0 \%$ \\
\hline Valor em falta & 15 & $14.3 \%$ \\
\hline \multicolumn{3}{|l|}{ Tipo de ensino } \\
\hline Regular & 21 & $20.0 \%$ \\
\hline Profissional & 7 & $6.7 \%$ \\
\hline Técnico & 56 & $53.3 \%$ \\
\hline Especial & 6 & $5.7 \%$ \\
\hline Valor em falta & 15 & $14.3 \%$ \\
\hline \multicolumn{3}{|l|}{ Retenções escolares } \\
\hline Nenhuma & 6 & $5.7 \%$ \\
\hline 1 & 12 & $11.4 \%$ \\
\hline 2 & 40 & $38.1 \%$ \\
\hline 3 ou mais & 47 & $44.8 \%$ \\
\hline \multicolumn{3}{|l|}{ Configuração familiar } \\
\hline Vivem com ambos os pais & 19 & $18.1 \%$ \\
\hline Famílias monoparentais & 16 & $15.2 \%$ \\
\hline Famílias reconstruídas & 7 & $6.7 \%$ \\
\hline $\begin{array}{l}\text { Encontram-se } \\
\text { institucionalizados }\end{array}$ & 57 & $54.3 \%$ \\
\hline Outra configuração & 6 & $5.7 \%$ \\
\hline
\end{tabular}




\begin{tabular}{lrr} 
Número de irmãos & & \\
0 & 2 & $1.9 \%$ \\
1 & 22 & $21.0 \%$ \\
2 & 28 & $26.7 \%$ \\
Mais de três irmãos & 46 & $43.8 \%$ \\
Valor em falta & 7 & $6.7 \%$ \\
\hline
\end{tabular}

Relativamente a características da família, verifica-se que 19 jovens habitam com ambos os pais (18.1\%), 16 vivem em famílias monoparentais $(15.2 \%)$ e mais de metade dos participantes encontram-se institucionalizados $(n=57 ; 54.3 \%)$. Salienta-se ainda que o número de irmãos é, em média, 3.07 $(D P=2.16)$.

\section{Instrumento}

Enquadrado num projeto internacional, o questionário MERLINO foi desenvolvido em parceria por investigadores de diferentes países da Europa. A versão portuguesa do questionário MERLINO (Matos et al., 2013 cit em Matos, Veríssimo, \& Soares, 2015) resultou da tradução da versão original e da sua adaptação através da introdução de novos itens para dar resposta aos objetivos específicos da equipa portuguesa. O processo de adaptação incluiu uma reflexão falada junto de sete jovens entre os 14 e os 17 anos.
O questionário MERLINO permite aceder a dimensões de risco e de proteção associadas à delinquência juvenil, sendo composto por 63 questões agrupadas em sete secções distintas: dados sociodemográficos e vivências pessoais; família e pessoas com quem vive; tempos livres; opinião sobre diferentes assuntos sociais; experiências de vitimação; atividades desviantes e ilícitas; e contactos com o sistema de justiça.

Para responder aos objetivos específicos deste estudo, foram selecionados os itens apresentados na tabela 2 .

\section{Procedimentos de Recolha e Análise de Dados}

Após autorização pela DGRSP, procedeu-se à recolha de dados entre fevereiro e junho de 2014 em centros educativos e equipas tutelares nas regiões de Lisboa e Porto. O questionário foi aplicado individualmente, na presença de um investigador, tendo sido salvaguardadas a confidencialidade dos dados e o anonimato dos participantes.

Tabela 2

Variáveis em Estudo: Variável Independente e Variáveis Dependentes

\begin{tabular}{lc}
\hline & Tipo de variável \\
\hline Variável independente & Nominal \\
Reincidência (número de contactos com o sistema de justiça) & Nominal \\
Variáveis dependentes & Nominal \\
História criminal de familiares & Ordinal \\
$\begin{array}{l}\text { Práticas educativas parentais (punição física, retirada de regalias, conversar, } \\
\text { ignorar) }\end{array}$ & (Escala de Likert) \\
Frequência de tempo passado com os pais (muito frequentemente a & Intervalar \\
frequentemente, às vezes, raramente ou nunca) & Nominal \\
Número de irmãos & Nominal \\
Testemunho de episódios de violência em casa & \\
Processo de promoção e proteção anterior &
\end{tabular}


Para análise dos dados, procedeu-se à recodificação da variável "contactos anteriores com o sistema de justiça", tendo sido criados três grupos: não reincidentes (um contacto com o sistema de justiça); reincidentes intermédios (de dois a dez contactos com o sistema de justiça); e, por fim, reincidentes persistentes (com mais de dez contactos com o sistema de justiça). Foi utilizado o teste de qui-quadrado para a análise da distribuição de frequências de variáveis nominais dependentes (e.g. história criminal de familiares) pelos diferentes grupos amostrais em análise (Hinton, 2004). Para analisar as diferenças na distribuição de variáveis dependentes ordinais (e.g., frequência de tempo que passa com os pais) assumindo a "reincidência" como variável independente (três grupos amostrais), foi utilizado o teste de Kruskal-Wallis, testando-se assim a distribuição das variáveis por três grupos amostrais distintos (Hinton, 2004; Maroco, 2007). Os dados recolhidos foram analisados com recurso ao software informático IBM SPSS Versão 22.

\section{Apresentação e Discussão dos Resul- tados}

Os resultados são apresentados e discutidos em duas secções principais, correspondentes aos dois objetivos definidos para o estudo. Assim, num primeiro momento, caracteriza-se o percurso judicial dos jovens em cumprimento de medida tutelar educativa e, em seguida, é analisada a associação entre os diferentes percursos de reincidência e as variáveis relativas à família e ao grupo de pares.
Caracterização do Percurso Judicial dos Jovens em Cumprimento de Medida Tutelar Educativa

Entre os tipos de crime que originaram a aplicação de medidas tutelares educativas, os crimes contra o património são os mais comuns, praticados por 68 jovens $(64.7 \%)$. Por outro lado, 55 jovens (52.3\%) cometeram pelo menos um crime contra as pessoas, e 15 jovens $(14.2 \%)$ um crime contra a vida em sociedade. De forma menos representativa, seis jovens $(5.7 \%)$ praticaram crimes enquadrados em legislação avulsa, cinco (4.8\%) praticaram crimes relacionados com estupefacientes e dois jovens (1.9\%) cometeram crimes contra o Estado. Enquanto 67 jovens $(63.4 \%)$ praticaram ilícitos criminais enquadrados somente num tipo de crime, $39(37.1 \%)$ cometeram crimes enquadrados em várias categorias. Entre estes jovens, a conjugação de "crimes contra as pessoas" e "contra o património" surge como a tipologia mais frequente $(n=18$; $17.1 \%)$.

No que se refere às medidas tutelares educativas em execução, 56 jovens (53.4\%) estão a cumprir medidas não institucionais ou na comunidade, enquanto 49 jovens estão a cumprir medidas institucionais ou de internamento $(46.7 \%)$. Focando as medidas não institucionais, 47 jovens estão em acompanhamento educativo (44.8\%), seguindo-se a imposição de obrigações, aplicada a 17 jovens $(16.2 \%)$. Verifica-se ainda que 10 jovens $(9.5 \%)$ estão a frequentar programas formativos, seis $(5.7 \%)$ estão a cumprir prestações económicas ou tarefas a favor da comunidade e um jovem (1.0\%) está a cumprir uma medida de reparação ao ofendido. 
Quanto à reincidência, 32 jovens são não reincidentes, tendo apenas um contacto com o sistema de justiça (30.5\%); 45 jovens $(41.9 \%)$ surgem como reincidentes intermédios (de dois a dez contactos com o sistema de justiça) e, de forma de menos expressiva, 23 participantes apresentam-se como reincidentes persistentes com mais de dez contactos com o sistema de justiça $(21.9 \%)$.

Entre as medidas tutelares educativas cumpridas anteriormente pelos jovens reincidentes, destacam-se o internamento em centro educativo (26 jovens; $24.8 \%$ ), o acompanhamento educativo (22 jovens; $21.0 \%$ ) e as prestações económicas ou tarefas a favor da comunidade (11 jovens; $10.5 \%)$.

A idade média de aplicação da primeira medida é de $14.58(D P=1.10)$. Dos 95 jovens em que foi possível apurar a precocidade da aplicação da primeira medida tutelar educativa, verifica-se que 56 jovens (58.9\%) foram alvo da primeira intervenção tutelar entre os 15 e os 17 anos de idade e 39 jovens $(41.1 \%)$ foram alvo da primeira medida entre os 12 e os 14 anos.

Família e Grupo de Pares em Jovens com Diferentes Percursos de Reincidência no Contacto com o Sistema de Justiça

Relativamente à família, a existência de processo de promoção e proteção anterior encontra-se associada à reincidência no contacto com o sistema de justiça, $\chi^{2}(2)=$ 21,$149 ; p<.001$. Tal como se verifica na tabela 3 , quase todos os jovens reincidentes persistentes tiveram um processo de promoção e proteção anterior $(91.3 \%)$, em comparação com $61.9 \%$ dos jovens do grupo de reincidentes intermédios e menos de um terço $(30.3 \%)$ dos jovens não reincidentes. Os dados parecem assim suportar a ideia de que quanto maior o número de contactos com o sistema de justiça, maior a probabilidade de o jovem já ter sido alvo de intervenção no âmbito da promoção e proteção. Tal ideia é compatível com as evidências de que os percursos persistentes de delinquência tendem a caracterizar-se pela conjugação de contactos "tutelares" e de proteção (Ryan et al., 2013; Stewart et al., 2002). A ocorrência na mesma trajetória de vida de contactos com o sistema de justiça juvenil e com o sistema de promoção e proteção leva-nos a questionar as possíveis formas de fragilidade familiar que lhe subjazem, assim como a própria dualidade do sistema, que providencia múltiplas respostas a esses jovens e às suas famílias em razão da sua natureza multiproblemática.

Os percursos de delinquência persistente parecem pautar-se por disrupção familiar, negligência, maltrato, ausência de suporte e de controle familiar na condenação de atos desviantes e delinquentes (Carvalho, 2005). Como refere Maria João Leote Carvalho, o fracasso do microssistema familiar reflete-se "numa multiplicidade de formas, em que a instabilidade, a violência recorrente e a ausência de figuras permanentes de referência constituem a imagem de marca" (2005, p. 90). Isto não significa necessariamente que as famílias dos jovens com percursos de reincidência persistente incentivem a prática de comportamentos delinquentes; antes, parecem ser incapazes de os inibir. Desta forma, admite-se que a família perde, em larga escala, a sua capacidade de disciplinar e de exercer controle social, especialmente durante a adolescência, etapa em que a suscetibilidade ao grupo de pares se intensifica (Içli \& Çoban, 2012). A este respeito, importa mencionar que as variáveis relativas às práticas educativas não se provaram estatisticamente associadas à reincidência. Pela faixa etária em que se encontram (14 aos 17 anos), estes jo- 
vens tendem a reclamar um afastamento mais acentuado em relação aos pais, passando o seu quotidiano a estruturar-se sobretudo em torno do grupo de pares. Nesse sentido, é possível que em fases desenvolvimentais mais precoces, as práticas educativas sejam mais influentes na adoção de comportamentos delinquentes (cf. Farrington, 2003).

Tabela 3

Frequências e Associações das Variáveis Dependentes em Estudo a Diferentes Percursos de Reincidência

\begin{tabular}{|c|c|c|c|c|c|c|c|}
\hline \multirow[b]{2}{*}{ Família } & \multicolumn{2}{|c|}{$\begin{array}{c}\text { Não } \\
\text { Reincidentes } \\
(n=35) \\
\end{array}$} & \multicolumn{2}{|c|}{$\begin{array}{l}\text { Reincidentes } \\
\text { intermédios } \\
(n=45) \\
\end{array}$} & \multicolumn{2}{|c|}{$\begin{array}{l}\text { Reincidentes } \\
\text { persistentes } \\
(n=23)\end{array}$} & \multirow[b]{2}{*}{$\chi^{2}(d f)$} \\
\hline & $n$ & $\%$ & $n$ & $\%$ & $n$ & $\%$ & \\
\hline História criminal & 15 & $42.9 \%$ & 27 & $61.4 \%$ & 14 & $60.9 \%$ & $3.12(2)$ \\
\hline Punição física & 6 & $18.2 \%$ & 7 & $16.3 \%$ & 1 & $4.5 \%$ & $2.25(2)$ \\
\hline Retirada de regalias & 17 & $51.5 \%$ & 18 & $41.9 \%$ & 7 & $31.8 \%$ & $2.12(2)$ \\
\hline Conversar & 24 & $72.7 \%$ & 32 & $74.4 \%$ & 12 & $54.5 \%$ & $2.96(2)$ \\
\hline Ignorar & 3 & $9.1 \%$ & 5 & $11.6 \%$ & 5 & $22.7 \%$ & $.58(2)$ \\
\hline Violência em casa & 5 & $16.7 \%$ & 11 & $25.6 \%$ & 5 & $22.7 \%$ & $.82(2)$ \\
\hline \multirow{2}{*}{$\begin{array}{l}\text { Processo de } \\
\text { promoção e proteção } \\
\text { anterior }\end{array}$} & 10 & $30.3 \%$ & 26 & $61.9 \%$ & 21 & $91.3 \%$ & $21.14(2)^{* *}$ \\
\hline & $M$ & $D P$ & $M$ & $D P$ & $M$ & $D P$ & $\chi^{2}(d f)$ \\
\hline Número de irmãos & 2.50 & 1.41 & 3.05 & 2.39 & 3.91 & 2.41 & $4.80(2)^{*}$ \\
\hline Tempo com os pais & $n$ & $\%$ & $n$ & $\%$ & $n$ & $\%$ & $\chi^{2}(d f)$ \\
\hline $\begin{array}{l}\text { Muito frequentemente } \\
\text { ou frequentemente }\end{array}$ & 20 & $57.1 \%$ & 18 & $41.9 \%$ & 15 & $65.2 \%$ & \multirow{3}{*}{$2.38(2)$} \\
\hline Às vezes & 9 & $25.7 \%$ & 14 & $32.6 \%$ & 4 & $17.4 \%$ & \\
\hline Raramente ou nunca & 6 & $17.1 \%$ & 11 & $25.6 \%$ & 4 & $17.4 \%$ & \\
\hline Grupo de pares & $n$ & $\%$ & $n$ & $\%$ & $n$ & $\%$ & $\chi^{2}(d f)$ \\
\hline Roubo em loja & 13 & $38.2 \%$ & 34 & $75.6 \%$ & 21 & $91.3 \%$ & $27.63(4)^{* *}$ \\
\hline $\begin{array}{l}\text { Roubo de dinheiro } \\
\text { ou objetos }\end{array}$ & 11 & $32.4 \%$ & 31 & $68.9 \%$ & 21 & $91.3 \%$ & $26.11(4)^{* *}$ \\
\hline Posse de arma & 8 & $24.2 \%$ & 21 & $47.7 \%$ & 18 & $78.3 \%$ & $16.61(4)^{* * *}$ \\
\hline Venda de drogas & 10 & $31.2 \%$ & 23 & $52.3 \%$ & 20 & $87.0 \%$ & $17.46(4)^{* *}$ \\
\hline $\begin{array}{l}\text { Roubo de carro ou } \\
\text { mota }\end{array}$ & 1 & $2.9 \%$ & 22 & $48.9 \%$ & 20 & $87.0 \%$ & $45.69(4)^{* *}$ \\
\hline $\begin{array}{l}\text { Condução sem } \\
\text { habilitação legal }\end{array}$ & 8 & $23.5 \%$ & 30 & $66.7 \%$ & 20 & $87.0 \%$ & $28.24(4)^{* *}$ \\
\hline
\end{tabular}


Questionando a atuação dual do sistema, compreende-se que a diversidade de problemáticas que caracterizam os percursos dos jovens com comportamento delinquente persistente se alia a uma multiplicidade de respostas formais, tipicamente compartimentadas, tanto por parte do sistema de justiça juvenil como do sistema de promoção e proteção. Por um lado, este intervencionismo pode ser motivado pelo maior escrutínio policial e pela atuação mais veemente do sistema de justiça em contextos de maior desvantagem social (Muncie, 2009). Por outro lado, a atuação dual e continuada pode revelar desarticulação e ineficácia dos serviços, que, em certa medida, poderão vitimizar secundariamente os jovens e perpetuar a sua permanência nos sistemas tutelar educativo e de proteção.

Não menos relevante para explicar a ligação entre a existência de um processo de promoção e proteção e a delinquência persistente, são os efeitos das experiências de maltrato (que motivam a instauração dos processos) no funcionamento individual dos jovens. A vivência (de forma direta ou vicariante) de maltrato pelos jovens pode promover a modelagem de características de funcionamento pessoal desajustadas, tais como a baixa tolerância à frustração (Loeber et al., 1998; Van der Laan, Veenstra, Bogaerts, Verhu1st, \& Ormel, 2010) e/ou a impulsividade (Loeber et al., 1998), frequentemente associadas à delinquência persistente. Estas características, tornando-se estáveis e típicas do funcionamento individual dos jovens, e sendo reforçadas socialmente, aumentam a probabilidade da ocorrência de comportamentos delinquentes persistentes. Esta hipótese explicativa dá ainda conta da possível interseção entre diferentes constelações de fatores de risco, neste caso fatores de ordem familiar, social e contextual (relacionados com a instaura- ção dos processos de promoção de proteção e as experiências de vitimação que estes traduzem) e individuais (assumidos nas repercussões discutidas a nível do funcionamento pessoal do jovem e da sua relação com o mundo).

No que respeita a história criminal da família de origem, ao contrário do que tem sido evidenciado na literatura, esta variável não se revelou associada à delinquência persistente. Uma possível explicação para este facto prende-se com o próprio desenho metodológico do presente estudo empírico. Enquanto os participantes foram inquiridos acerca da existência de história criminal em toda a família, tanto nuclear como alargada, os estudos que mostram que a história criminal e/ou de reclusão da família de origem é preditora da delinquência juvenil centram-se sobretudo na família nuclear (pais ou irmãos) (cf. Farrington, 2003; Murray \& Farrington, 2005).

Relativamente ao grupo de pares, todos os comportamentos elencados na tabela 3 estão associados à reincidência, designadamente o roubo em loja, $\chi^{2}(4)=27,634 ; p<$ .001 ; o roubo de dinheiro ou outros objetos $\chi^{2}(4)=26,111 ; p<.001$; a posse de arma, $\chi^{2}(4)=16,691 ; p<.001 ;$ a venda de drogas $\chi^{2}(4)=17,462 ; p<.001 ;$ o roubo de carro ou mota, $\chi^{2}(4)=45,693 ; p<.001$; e, por fim, a condução sem habilitação legal $\chi^{2}$ (4) $=28,241 ; p<.001$. Os jovens com percursos de reincidência persistente assumem mais frequentemente possuir amigos que já perpetraram essas ofensas, em comparação com jovens com percursos de reincidência intermédia ou não reincidentes. Por outro lado, um percurso caracterizado por elevada persistência a nível de comportamentos delinquentes, saturado pela presença de pares desviantes, pode dificultar a afiliação a pares normativos, o que aumenta também o risco de delinquência (Vitaro et al., 2005). 
A ausência de modelos normativos nas redes de pares e a maior suscetibilidade para ser influenciado pelo grupo de pares desviantes (Miller, 2010), poderá explicar, em parte, a reincidência criminal.

\section{Conclusão}

Este estudo pretendeu conhecer o percurso judicial de jovens em cumprimento de medida tutelar educativa e compreender como algumas características da família e do grupo de pares estão associadas à sua reincidência nos contactos com o sistema de justiça. A partir da análise dos dados conclui-se, antes de mais, que algumas dimensões familiares e do grupo de pares estão de facto associadas a diferentes níveis de reincidência em trajetórias de jovens sinalizados por comportamento delinquente.

Quando analisamos os percursos de jovens com comportamentos delinquentes persistentes, percebemos que eles são expostos a múltiplas formas de vulnerabilidade social, desde logo na família, provindo de contextos familiares mais frequentemente sinalizados pelo sistema de promoção e proteção.

A exposição a diferentes formas de maltrato e de marginalidade nos seus contextos relacionais mais imediatos (família, grupo de pares) pode proporcionar aos jovens um ambiente legitimador da delinquência a partir de redes sociais mais amplas, que conduz à normalização e à aceitação da delinquência (cf. HallidayBoykins \& Graham, 2001; Içli \& Çoban, 2012). Esta evidência confirma o argumento de que a violência é uma questão sociocultural, internalizada nos discursos dos jovens pertencentes a contextos multiproblemáticos, que condensam problemas de natureza familiar, relacional e sociocultural (Haynie, Silver, \& Teasdale, 2006).
Esses elementos apontam para situações de elevada vulnerabilidade social, estando estes jovens sujeitos a maior e mais precoce intervencionismo social e judicial, representado neste estudo pelos contactos duais entre os sistemas de justiça juvenil e de proteção. Ao possuírem menor número de modelos familiares e sociais orientados para a adoção de comportamentos prossociais e ditos convencionais, é provável que no momento em que intervenção cessa, os principais fatores associados à persistência do comportamento delinquente perdurem.

Em síntese, as dimensões familiares e associadas ao grupo de pares analisadas neste estudo podem contribuir para explicar a persistência na adoção de comportamentos delinquentes após sucessivas intervenções formais. As múltiplas intervenções a que as crianças e jovens são sujeitos podem nem sempre significar maior proteção e investimento na sua (re)educação. Entendemos, por isso, que a criação de condições práticas com vista à monitorização dos percursos dos jovens após a passagem pelo sistema tutelar educativo pode ser importante. Deve também ser evitada a fragmentação de respostas e promovida uma intervenção mais integrada, que atue ao nível dos múltiplos problemas que parecem pautar a vida dos jovens reincidentes.

Por fim, é relevante discutir as principais limitações do estudo e apresentar propostas de investigação futura. Como limitação, referimos a análise do comportamento delinquente em jovens entre os 14 e os 17 anos inferindo acerca da reincidência através de um desenho metodológico retrospetivo. Para avaliar a reincidência seria pertinente o desenvolvimento de um estudo empírico longitudinal, que permitisse aceder à evolução do comportamento delinquente durante a adolescência e a 
passagem para a vida adulta. Através de um desenho longitudinal seria ainda possível avaliar diferentes tipos de percursos de reincidência, considerando a evolução, a severidade e o tipo de crimes praticados (cf. Mulder et al., 2012; Van Domburgh et al., 2009). Também a constituição da amostra pode ser apontada como limitação, na medida em que não é representativa do universo de jovens com medida tutelar educativa em Portugal. Em estudos futuros seria importante abranger mais jovens em cumprimento de medidas na comunidade, tais como tarefas a favor da comunidade e imposição de obrigações, e considerar maior diversidade de crimes que motivaram a aplicação da medida tutelar educativa.

Ainda em relação a investigação futura, consideramos importante a análise de outros preditores da reincidência (e.g. de natureza individual - sexo, competências sociais - escolar - competências escolares, envolvimento escolar - e, ainda, contextual - violência na vizinhança, nível socioeconómico), assim como uma abordagem mais sistematizada e integrada dos fatores discutidos neste artigo, recorrendo a diferentes indicadores, medidas e análises. Consideramos também fundamental perceber que fatores promovem a desistência em percursos de delinquência persistente. Sabendo já que determinados fatores protetores, por exemplo familiares, estão relacionados com o abandono de comportamentos delinquentes (Van Domburgh et al., 2009), será fundamental conhecer as especificidades e o modo como atuam os fatores que promovem a desistência em percursos delinquentes caracterizados pela persistência.

\section{Referências}

Andersson, F., Levander, S., Svensson, R., \& Levander, M. (2012). Sex differences in offending trajectories in a Swedish cohort. Criminal Behavior and Mental Health, 22(2), 108-121. http://dx.doi.org/10.1002/cbm.1822

Ang, R. \& Huan, V. (2008). Predictors of recidivism for adolescent offenders in a Singapore sample. Criminal Justice and Behavior, 35(7), 895-905. http://dx.doi.org/10.1177/0093854808317270

Aseltine, R. H. (1995). A reconsideration of parental and peer influences on adolescent deviance. Journal of Health and Social Behavior, 36(2), 103-121.

Carvalho, M. (2005). Jovens, espaços, trajectórias e delinquências. Sociologia, Problemas e Práticas, 49, 71-93.

Chung, L. \& Steinberg, L. (2006). Relations between neighborhood factors, parenting behaviors, peer deviance, and delinquency among serious juvenile offenders. Developmental Psychology, 42(2), 319-331. http://dx.doi.org/10.1037/0012-1649.42.2.319

Church II, W., Tomek, S., Bolland, K., Hooper, L., Jaggers, J., \& Bolland, J. (2012). A longitudinal examination of predictors of delinquency: An analysis of data from the Mobile Youth Survey. Children and Youth Services Review, 34(12), 2400-2408. http://dx.doi.org/10.1016/j.childyouth.2012.09.007 
Contreras, L., Molina, V., \& Cano, M. C. (2011). In search of psychosocial variables linked to the recidivism in young offenders. The European Journal of Psychology Applied to Legal Context, 3(1), 77-88.

Cottle, C., Lee, R., \& Heilbrun, K. (2001). The prediction of criminal recidivism in juveniles: A meta-analysis. Criminal Justice and Behavior, 28(3), 367-394. http://dx.doi.org/10.1177/0093854801028003005

Crooks, C., Scott, K., Wolfe, D., Chiodo, D., \& Kilip, S. (2007). Understanding the link between childhood maltreatment and violent delinquency: What do schools have to add? Child Maltreatment, 12(3), 269-280.

http://dx.doi.org/10.1177/1077559507301843

Day, D., Wanklyn, S., \& Yessine, A. (2014). A review of terminological, conceptual, and methodological issues in the developmental risk factor literature for antisocial and delinquent behavior. Child Youth Care Forum, 43(1), 97-112.

http://dx.doi.org/10.1007/s10566-013-9227-9

Direção Geral de Reinserção e Serviços Prisionais, DGRSP (2014). Síntese estatística Reinserção Social outubro 2014. Retrieved from

http://www.dgrs.mj.pt/web/rs/estat

Dishion, T. \& Patterson, G. (2006). The development and ecology of antisocial behavior in children and adolescents. In D. Cicchetti \& D. Cohen (Eds.), Developmental psychopathology (pp. 503-541). New Jersey: John Wiley \& Sons.

Farrington, D. (2003). Key results from the first forty years of the Cambridge study in delinquent development. In T. Thornberry \& M. Krohn (Eds.), Taking stock of delinquency: An overview of findings from contemporary longitudinal studies (pp. 137-183). New York: Kluwer/Plenum.

Farrington, D. (2010). Family influences on delinquency. In D. Springer \& R. Roberts (Eds.), Juvenile justice and delinquency (pp. 203-222). Sudbury: Jones and Bartlett.

Farrington, D., Loeber, R., \& Ttofi, M. (2012). Risk and protective factors for offending. In B. C. Welsh \& D. P. Farrington (Eds.), The Oxford handbook of crime prevention (pp. 46-69). Oxford, UK: Oxford University Press.

Farrington, D. \& Ttofi, M. (2011). Protective and promotive factors in the development of offending. In T. Bliesener, A. Beelmann, \& M. Stemmler (Eds.). Antisocial behavior and crime: Contributions of developmental and evaluation research to prevention and intervention (pp. 71-88). Cambridge: Hogrefe Publishing.

Fergusson, D., Vitaro, F., Wanner, B., \& Brendgen, M. (2007). Protective and compensatory factors mitigating the influence of deviant friends on delinquent behaviours during early adolescence. Journal of Adolescence, 30(1), 33-50.

http://dx.doi.org/10.1016/j.adolescence.2005.05.007

Fréchette, M. \& Le Blanc, M. (1987). Délinquances et délinquants. Québec: Gaetan Morin. 
Gardner, M. \& Steinberg, L. (2005). Peer influence on risk taking, risk preference, and risky decision making in adolescence and adulthood: An experimental study. Developmental Psychology, 41(4), 625-635.

http://dx.doi.org/10.1037/0012-1649.41.4.625

Griffin, K., Botvin, G., Scheier, L., Diaz, T., \& Miller, N. (2000). Parenting practices as predictors of substance use, delinquency, and aggression among urban minority youth: Moderating effects of family structure and gender. Psychology of Addictive Behaviors, 14(2), 174-184.

http://dx.doi.org/10.1037//0893-164X.14.2.174

Halliday-Boykins, C. \& Graham, S. (2001). At both ends of the gun: Testing the relationship between community violence exposure and youth violent behavior. Journal of Abnormal Child Psychology, 29(5), 383-402.

Hartinger-Saunders, R., Rine, C., Wieczorek, W., \& Nochajski. T. (2012). Family level predictors of victimization and offending among young men: Rethinking the role of parents in prevention and interventions models. Children and Youth Services Review, 34(12), 2423-2432.

http://dx.doi.org/10.1016/j.childyouth.2012.08.017

Haynie, D. \& Payne, D. (2006). Race, friendship networks, and violent delinquency. Criminology, 44(4), 775-805.

http://dx.doi.org/ 10.1111/j.1745-9125.2006.00063.x

Haynie, D., Silver, E., \& Teasdale, B. (2006). Neighborhood characteristics, peer networks, and adolescent violence. Journal of Quantitative Criminology, 22(2), 147-169.

http://dx.doi.org/10.1007/s10940-006-9006-y

Hinton, P. (2004). Statistics explained ( $2^{\text {nd }}$ Ed.). New York: Taylor and Francis.

Hoeve, M., Blokland, A., Dubas, J., Loeber, R., Gerris, I., \& Van der Laan, P. (2007). Trajectories of delinquency and parenting styles. Journal of Abnormal Child Psychology, 36(2), 223-235.

http://dx.doi.org/10.1007/s10802-007-9172-x

Hoeve, M., Dubas, J. S., Eichelsheim, V. I., Van der Laan, P. H., Smeenk, W., \& Gerris, J. R. M. (2009). The relationship between parenting and delinquency: A meta-analysis. Journal of Abnormal Child Psychology, 37(6), 749-775.

http://dx.doi.org/10.1007/s10802-009-9310-8

Huizinga, D., Weiher, A., Menard, S., Espiritu, R., \& Esbensen, F. (november, 1998). Some not so boring findings from the Denver Youth Survey. Paper presented at the American Society of Criminology Meeting, Washington, DC.

Içli, T. \& Çoban, S. (2012). A study on the effects of family and delinquent peers on juvenile delinquency in Turkey. Advances in Applied Sociology, 2(1), 66-72.

http://dx.doi.org/10.4236/aasoci.2012.21009

Ireland, T., Smith, C., \& Thornberry, T. (2002). Development issues in the impact of child maltreatment on later delinquency and drug use. Criminology, 40(2), 359-400.

http://dx.doi.org/10.1111/j.1745-9125.2002.tb00960.x 
Kazemian, L. \& Farrington, D. (2005). Comparing the validity of prospective, retrospective, and official onset for different offending categories. Journal of Quantitative Criminology, 21(2), 127-147.

http://dx.doi.org/10.1007/s10940-005-2489-0

Le Blanc, M. \& Fréchette, M. (1989). Male criminal activity from childhood through youth: Multilevel and developmental perspectives. New York: Springer.

Lee, J., Onifade, E., Teasley, M., \& Noel, L. (2012). The effects of risk and protective factors on juvenile delinquency in South Korea. Journal of Ethnicity in Criminal Justice, 10(4), 316-329.

http://dx.doi.org/10.1080/15377938.2012.732880

Lei $N^{o} 4 / 2015$ de 15 de Janeiro de 2015. Diário da República $N^{o} 10-1^{\text {a }}$ Série-A. Assembleia da República: Lisboa.

Lei $N^{o} 147 / 99$ de 1 de Setembro de 1999. Diário da República $N^{o} 204-1^{\text {a }}$ Série-A. Assembleia da República: Lisboa.

Lei $N^{o} 166 / 99$ de 14 de Setembro de 1999. Diário da República $N^{o} 215$ - $1^{\text {a }}$ Série-A. Assembleia da República: Lisboa.

Loeber, R., Farrington, D. P., Stouthamer-Loeber, M., Moffitt, T., \& Caspi, A. (1998). The development of male offending: Key findings from the first decade of the Pittsburgh Youth Study. Studies on Crime and Crime Prevention, 7, 141-172.

Maroco, J. (2007). Análise estatística - com a utilização do SPSS ( $3^{\mathrm{a}}$ Ed.). Lisboa: Edições Sílabo.

Matos, R., Veríssimo, L., \& Soares, M. (2015). Portugal - National analysis (Relatório Final do projeto MERLINO “Towards a juvenile delinquency prevention policy: a multistate experiment on integration and efficacy" HOME/2011/ISEC/AG/4000002611). Porto: Universidade Católica Portuguesa.

Miller, H. (2010). If your friends jumped off a bridge, would you do it too? Delinquent peers and susceptibility to peer influence. Justice Quarterly, 27(4), 473-491.

http://dx.doi.org/10.1080/07418820903218974

Moffitt, T. (1993). Adolescence-limited and life-course-persistent antisocial behaviour: A developmental taxonomy. Psychological Review, 100, 674-701.

Moffitt, T. (2006). Life-course-persistent versus adolescence-limited antisocial behavior. In D. Cicchetti \& D. Cohen (Eds.), Developmental psychopathology (pp. 570-598). New Jersey: Wiley.

Morris, S. \& Gibson, C. (2011). Corporal punishment's influence in children's aggressive and delinquent behavior. Criminal Justice and Behavior, 38(8), 818-839.

http://dx.doi.org/10.1177/0093854811406070

Mulder, E., Vermunt, J., Brand, E., Bullens, R., \& Van Marle, H. (2012). Recidivism in subgroups of serious juvenile offenders: Different profiles, different risks? Criminal Behavior and Mental Health, 22(2), 122-135.

http://dx.doi.org/10.1002/cbm.1819

Muncie, J. (2009). Youth and crime ( $3^{\mathrm{a}}$ Ed.). California: Sage Publications. 
Murray, J. \& Farrington, D. (2005). Parental imprisonment: Effects on boys' antisocial behaviour and delinquency through the life-course. Journal of Child Psychology and Psychiatry, 46(12), 1269-1278.

http://dx.doi.org/10.1111/j.1469-7610.2005.01433.x

Ryan, J., Williams, A., \& Courtney, M. (2013). Adolescent neglect, juvenile delinquency and the risk of recidivism. Journal of Youth Adolescence, 42(3), 454-465.

http://dx.doi.org/10.1007/s10964-013-9906-8

Shader, M. (2003). Risk factors for delinquency: An overview. Office of Juvenile Justice and Delinquency Prevention. Retrieved from

https://www.ncjrs.gov/html/ojjdp/jjjournal_2003_2/index.html

Simões, C., Matos, M. G., \& Batista-Foguet, J. M. (2008). Juvenile delinquency: Analysis of risk and protective factors using quantitative and qualitative methods. Cognition, Brain, Behavior: An Interdisciplinary Journal, 12(4), 389-408.

Smith, C., Ireland, T., \& Thornberry, T. (2005). Adolescent maltreatment and its impact on young adult antisocial behavior. Child Abuse \& Neglect, 29(10), 1099-1119.

http://dx.doi.org/10.1016/j.chiabu.2005.02.011

Smith, C. \& Thornberry, T. (1995). The relationship between childhood maltreatment and adolescent involvement in delinquency. Criminology, 33(4), 451-481.

Sousa Santos, B. (2004). Os caminhos difíceis da "nova" justiça tutelar educativa - uma avaliação de dois anos de aplicação da Lei Tutelar Educativa. Coimbra: Centro de Estudos Sociais.

Stattin, H. \& Kerr, M. (2000). Parental monitoring: A reinterpretation. Child Development, 71(4), 1072-1085.

http://dx.doi.org/10.1111/1467-8624.00210

Stewart, A., Dennison, S., \& Waterson, E. (2002). Pathways from child maltreatment to juvenile offending. Research report from Criminology Research Council. Retrieved from Australian Criminology Research Grants:

http://crg.aic.gov.au/reports/200304-35.pdf

Stouthamer-Loeber, M., Loeber, R., Stallings, R., \& Lacourse, E. (2008). Desistance from and persistence in offending. In R. Loeber, D. Farrington, M. Stouthamer-Loeber, H. R. White, \& E. Wei (Eds.), Violence and serious theft: Development and prediction from childhood to adulthood (pp. 269-308). New York: Routledge.

Stouthamer-Loeber, M., Loeber, R., Wei, E., Farrington, D., \& Wikström, P. (2002). Risk and promotive effects in the explanation of persistent serious delinquency in boys. Journal of Consulting and Clinical Psychology, 70(1), 111-123.

Thomas, K. (2015). Delinquent peer influence on offending versatility: Can peers promote specialized delinquency? Criminology, 53(2), 280-308.

http://dx.doi.org/10.1111/1745-9125.12069

Thornberry, T. P., Krohn, M. D., Lizotte, A. J., Smith, C. A., \& Porter, P. K. (1998). Taking stock: An overview of findings from the Rochester youth development study. American Society of Criminology, 12, 1-53. 
van der Laan, A., Veenstra, R., Bogaerts, S., Verhulst, F., \& Ormel, J. (2010). Serious, minor and non-delinquents in early adolescence: The impact of cumulative risk and promotive factors: The TRAILS study. Journal of Abnormal Child Psychology, 38(3), 339351.

http://dx.doi.org/10.1007/s10802-009-9368-3

van Domburgh, L., Loeber, R., Bezemer, D., Stallings, R., \& Stouthamer-Loeber, M. (2009). Childhood predictors of desistance and level of persistence in offending in early onset offenders. Journal of Abnormal Child Psychology, 37(7), 967-980. http://dx.doi.org/10.1007/s10802-009-9329-X

Vitaro, F., Brendgen, M., \& Wanner, B. (2005). Patterns of affiliation with delinquent friends during late childhood and early adolescence: Correlates and consequences. Social Development, 14(1), 82-108.

http:dx.doi.org/ 10.1111/j.1467-9507.2005.00292.x 